\title{
TTR
}

Traduction, terminologie, re?daction

\section{L'Influence des religions catholique et protestante sur la traduction des textes sacrés à l'intention des Micmacs dans les provinces Maritimes : du livre de prières de l'abbé Maillard (1710-1762) à la traduction des Évangiles par Silas Tertius Rand (1810-1889)}

\section{Christel Gallant}

Volume 3, numéro 2, 2e semestre 1990

La traduction des textes sacrés : le domaine biblique

URI : https://id.erudit.org/iderudit/037071ar

DOI : https://doi.org/10.7202/037071ar

Aller au sommaire du numéro

Éditeur(s)

Association canadienne de traductologie

ISSN

0835-8443 (imprimé)

1708-2188 (numérique)

Découvrir la revue

Citer cet article

Gallant, C. (1990). L'Influence des religions catholique et protestante sur la traduction des textes sacrés à l'intention des Micmacs dans les provinces Maritimes : du livre de prières de l'abbé Maillard (1710-1762) à la traduction des Évangiles par Silas Tertius Rand (1810-1889). TTR, 3(2), 97-109. https://doi.org/10.7202/037071ar 


\section{L'Influence des religions catholique et protestante sur la traduction des textes sacrés à l'intention des Micmacs dans les provinces Maritimes: du livre de prières de l'abbé Maillard (1710-1762) à la traduction des Évangiles par Silas Tertius Rand (1810-1889)}

\section{Christel Gallant}

\section{Introduction}

On pourrait penser que l'expérience canadienne n'a pas grand-chose à apporter à l'étude de la traduction des textes sacrés, car le pays n'est pas le berceau d'une grande religion et sa population de souche européenne se compose essentiellement de catholiques et protestants - francophones ou anglophones - qui ont toujours été tributaires des traductions religieuses faites dans leurs pays d'origine respectifs, ressemblant en cela aux Canadiens issus d'autres parties du globe et d'autres univers religieux.

Pourtant, dans le sillage de la conquête et de la colonisation du Canada actuel par ce qu'il est convenu d'appeler les «deux peuples fondateurs», il y eut non seulement des tentatives répétées de christianisation auprès des "premières nations», mais encore un important effort de traduction vers les langues autochtones.

La particularité de cette activité traduisante réside dans son articulation chronologique en deux phases: une première, catholique 
et française, et une deuxième, protestante et essentiellement anglaise.

Si toutes les régions et toutes les nations du Canada n'ont pas été touchées par les deux vagues successivement, tel est cependant le cas des Amérindiens d'Acadie. Colonie peuplée de paysans de souche française depuis la fondation de Port-Royal en 1604, objet de plusieurs coups de main et restitutions successifs, l'Acadie est passée définitivement du régime français au régime anglais en 1713, mais elle n'a connu un influx important de colons protestants, et le plus souvent anglophones, qu'à partir de la fondation d'Halifax en 1749, renforcé encore après la déportation des Acadiens en 1755 et par l'arrivée des Loyalistes après la Révolution américaine.

La population indigène de cette grande région - désormais disparue des cartes politiques au profit des trois provinces Maritimes de la Nouvelle-Ecosse, de l'lle-du-Prince-Edouard et du Nouveau-Brunswick - a toujours été très homogène. A l'exception de la vallée de la rivière Saint-Jean au Nouveau-Brunswick, territoire des Malécites, c'est le pays des Micmacs, qui englobe d'ailleurs aussi une partie de la péninsule gaspésienne au Québec. Les Micmacs, qui appartiennent comme les Malécites à la grande famille algonquienne, représentent aujourd'hui environ quatre pour cent du demi million d'Amérindiens canadiens.

Au cours des presque trois siècles écoulés entre l'arrivée des premiers colons blancs et français en Acadie et la généralisation de l'anglais comme langue véhiculaire par une politique de scolarisation obligatoire, le refus des Micmacs d'apprendre une autre langue que la leur et le recours à l'interprétation dans toutes les tractations entre Amérindiens et colons ou administrateurs de souche européenne constituent un aspect particulièrement intéressant de l'histoire de la traduction en Acadie.

Il est donc important de noter que les travaux de deux missionnaires, l'abbé Maillard au $18^{\mathrm{e}}$ siècle et le pasteur Rand au $19^{\circ}$, à qui nous devons à la fois d'importantes descriptions linguistiques et les traductions qui font l'objet de cette communication, ne représentent qu'une facette parmi d'autres du contact forcé entre les trois groupes linguistiques qui cohabitent encore aujourd'hui dans les Maritimes.

Il ne s'agit pas pour autant de simples faits divers. Aussi bien Silas Rand, le pasteur baptiste, que, avant lui, l'abbé Maillard 
s'inscrivent, chacun pour sa religion, dans un cadre d'évangélisation plus général et relativement bien établi, ce qui permet de penser qu'en faisant les recherches appropriées on trouvera ailleurs au Canada d'autres médiateurs-traducteurs sinon de leur trempe, du moins comparables.

Aux traductologues intéressé(e)s, le livre de John Webster Grant, Moon of Wintertime, ${ }^{1}$ même s'il n'accorde aucune attention particulière à l'aspect traductionnel de la mission, pourrait sans doute servir de guide pour découvrir d'autres exemples des préférences respectives des missionnaires catholiques et protestants quant aux choix des textes à traduire dans le cadre de leur apostolat.

\section{Maillard et la traduction catholique}

Pierre Maillard, prêtre des Missions étrangères, est né en France dans le diocèse de Chartres vers 1710 et décédé en NouvelleÉcosse, à Halifax, le 12 août $1762 .^{2}$

Quand il arrive dans la mission micmaque de l'île Royale, restée française (aujourd'hui l'île du Cap-Breton) en 1735, son intervention a été bien préparée sur deux plans: il a reçu un encadrement rigoureux au séminaire du Saint-Esprit à Paris et il bénéficie des fruits d'un peu plus d'un siècle de missions françaises auprès des Micmacs.

Les missionnaires qui le précèdent, ou qui œuvrent en même temps que lui auprès d'autres groupes de Micmacs, ont depuis longtemps dépassé le stade de la première exploration pénible et rudimentaire du lexique micmac, décrite avec tant de verve par le père Biard dans sa Relation de la Nouvelle-France 1616 au chapitre XVI qui traite, entre autres, de «la difficulté d'apprendre la langue des Sauvages». Entre-temps, ils ont le plus souvent assez bien appris la langue par immersion et avec l'aide de métis bilingues ou bien grâce à une période d'initiation auprès de collègues déjà sur place. Quant à la situation religieuse, l'abbé

1. John Webster Grant, Moon of Wintertime: Missionaries and Indians of Canada in Encounter since 1534 (Toronto, University of Toronto Press, 1984).

2. Cf. Dictionnaire biographique du Canada, vol. III (Québec et Toronto, Les Presses de 1'Université Laval, 1974) pp. 448-452. 
Gaulin affirme qu'en 1710 , un siècle donc après le premier baptème micmac administré au chef Membertou et à une vingtaine de membres de sa tribu par le père Jessé Fléché, tous les Micmacs ont embrassé la foi catholique qu'ils continuent à pratiquer sous le régime anglais.

Il ne s'agit donc plus d'obtenir des conversions mais d'instruire et de maintenir les communautés micmaques dans la religion catholique. Il importe aussi de les armer contre l'hérésie protestante et d'utiliser cet antagonisme spirituel à des fins bien prosaïques: s'assurer de la loyauté des Micmacs au Roi Très Chrétien et donner à leur collaboration avec les troupes françaises de l'Ile Royale ou de Québec des accents de guerre sainte. Cet aspect temporel de la mission aux confins d'une Acadie cédée aux Anglais mais toujours convoitée, n'est pas sans importance, car il renforce encore davantage l'attitude tutélaire qui caractérise souvent l'Église catholique.

Parallèlement à ses devoirs de prêtre et de Français, Maillard, qui possède des dons linguistiques exceptionnels, très prisés des Micmacs, s'attache surtout à la consolidation du savoir linguistique et à sa transmission structurée à ses successeurs, en composant une importante grammaire manuscrite, recopiée en plusieurs exemplaires par la suite et qui témoigne de sa grande maîtrise de cette langue difficile dont il a patiemment et minutieusement exploré les structures.

Il se consacre aussi à la consolidation de l'instruction religieuse des Micmacs, qui nous intéresse plus particulièrement ici, puisqu'elle a donné lieu aux traductions religieuses proprement dites. Celles-ci sont de deux sortes, ou plus exactement prennent deux formes distinctes, selon qu'elles sont destinées à ses confrères ou à ses ouailles.

Pour la transcription du micmac à l'intention du clergé, le père Maillard utilise, comme dans ses ouvrages grammaticaux, l'alphabet latin légèrement modifié. Ses traductions se présentent sous forme de cahiers bilingues avec texte français en regard du texte micmac.

A l'usage des Micmacs eux-mêmes, il utilise une notation hiéroglyphique, largement de sa propre invention, qu'il leur fait apprendre et copier dans des cahiers personnels, recopiés par la suite à de multiples reprises. On trouvera dans les illustrations des 
exemples - imprimés ultérieurement - à la fois des hiéroglyphes et des textes en écriture alphabétique.

Les traductions transcrites en hiéroglyphes, ou idéogrammes, comprennent quelques textes tirés directement de la Bible, comme l'oraison dominicale, la salutation angélique, la profession de foi apostolique ou le décalogue, et, dans une proportion bien plus importante, des textes d'instruction religieuse, comme les catéchismes (général, pour enfants, ou encore de la communion) ou les sacrements, les "vérités de la religion», des prières, des cantiques, des passages liturgiques ainsi qu'un condensé d'une partie de l'histoire sainte dont une note indique qu'il est tout entier tiré du «catéchisme historique», sans doute celui de Claude Fleury, paru à Paris en 1683.

Il s'agit d'un cas de traduction fonctionnant parfaitement dans la civilisation d'accueil, car même après la mort du père Maillard et en l'absence de missionnaires catholiques résidents pendant près d'un siècle, ces traductions ont été soigneusement conservées, mémorisées, recopiées et transmises d'une génération à l'autre. Leur utilisation prolongée et le prestige qu'elles avaient acquis auprès des Micmacs en font un fait culturel et un facteur de cohésion comparables, toute proportion gardée, à certaines grandes traductions européennes de la Bible du $16^{\circ}$ siècle, comme la Bible allemande de Luther ou la Bible tchèque des frères moraves.

Et on peut se demander si, grâce à leur qualité, grâce à leur présentation idéographique fortement symbolique et sans doute aussi grâce à l'extraordinaire ascendant du père Maillard sur les Micmacs qui semblaient croire à une origine surnaturelle ou inspirée de ses connaissances linguistiques, ces traductions n'ont pas accédé elles-mêmes au statut de texte sacré. La redécouverte de ces cahiers dans la deuxième moitié du $19^{\circ}$ siècle par le missionnaire luxembourgeois Kauder a finalement abouti à une première édition à Vienne en 1866, malheureusement presque entièrement disparue dans un naufrage, et dont un des rares exemplaires existants peut être consulté dans la collection des livres extrêmement rares des Archives publiques de la Nouvelle-Écosse. ${ }^{3}$ Comme la reproduction

3. Public Archives of Nova Scotia (PANS), «Picture word» edition of the Roman catholic catechism (Vienna 1866) cote : AK-F17.C28. 
Credo - 'The Apostles' Creed

\section{$\gg \sqrt{1}$ 每}

o>

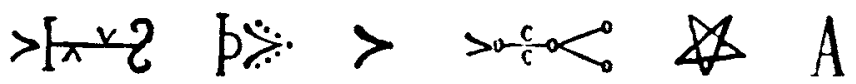

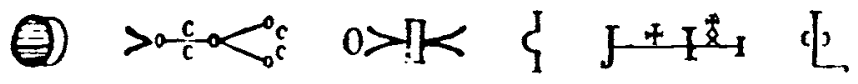

小 $p$

a 30 N: $\mathbb{N}^{*}>R_{1} X>B$

$>{ }^{*} \mathrm{C}>\mathrm{F}^{\mathrm{\gamma}} \otimes \mathrm{m}$



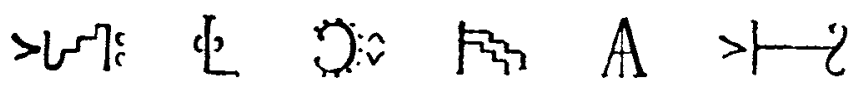

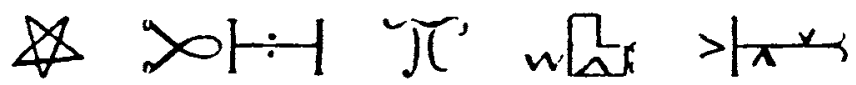

$*$ RR A 2ens o>T \& $\triangle$ 
Ave Maria.

MALI oesgagelmol, gil sapeotil mestanemenel, Gsagmamino geogonsg gtinin metj tegoeiat, neogtetjin oelein epitjig mo telcionig. gois Sesos msegilteo gionagaij emtógoalot.

Gisna.

GoleIn, Mali, sapeotil oatjopinel,

Gsagmamino tegoeiasg, epitjig patjitji oleinig, Ag oeleg tan gtelamilog

Oetjimanit Sesos.

Petsili sapeoin Mali oegoisin Oestaolgop,

Nigetj alasotmelseoin eloeoltieg,

Ag apis oigoiatieg

Alasotmelseoitesnen.

Credo.

1. Oeli getlamsetasi Gtjinisgam Oegoisit mset gôgoel tan telitetgel telatigel," netna negem gisitôgsep oasûg ag magamigeo.

2. Oeli getlamsetasi elp Sesogoli ogoisel neog. tigilitjel "netna negem oesagmamoltigo.

3. Oetjioli Nisgam piptógópsgalasenel, elnoalasenel, " gesg Mali nasgoeteg ogtelamilog oetji osgitjinoilisenel.

4. Amasgoipelnotag Pons Pilatôg sagmaoiteg," glotjieogtôtag, nepgag, otgota lotag.

5. Lamgamog elipgeoietag," tapogonageg oetji minonsiseni nepelipeni.

6. Oasôg oli onagietag, " otjel ogtinagang mset gôgoel tan telitetgel telatigel epitjel.

7. Aptj pgisinteo, oasóg ogtjieteo tan telitelmatj telelitag oesgitjinoliilitji ag iaptji nelitji.

8. Oenr getlamsetasi * Oetjioli Nisgam.

9. Santeoi mesta Maoiómi," sapeotil maoiatj.

10. Eloeotil meniatj.

11. Mageil apitjipetal. *

12. laptji osgitjinoti. Amen. 
n'en est pas autorisée, l'illustration ici présentée provient de la réédition de l'ouvrage réalisée en $1921{ }^{4}$

Quant à la version en écriture alphabétique, destinée aux autres missionnaires, elle comporte, en plus de ces mêmes textes, divers offices et développements liturgiques ainsi que quelques exemples d'exhortations ou prônes. Elle constitue, de son côté, la base du catéchisme micmac en écriture alphabétique, imprimé en 1913, puis de nouveau en 1921, après avoir été augmentée considérablement par le père capucin Pacifique de Valigny, ${ }^{5}$ grand spécialiste des Micmacs au $20^{\circ}$ siècle.

Mais avant le retour en force du clergé catholique, les Micmacs, qui sont passés dans leur totalité sous autorité anglaise depuis la chute définitive de Louisbourg, le traité de Paris et leurs traités de paix avec le gouvernement à Halifax, font l'objet de diverses tentatives de conversion au protestantisme, dont la plus importante, celle de Silas Tertius Rand, est à l'origine d'un autre groupe de traductions, cette fois-ci entièrement bibliques.

\section{Rand et la traduction protestante}

Le deuxième grand traducteur de textes sacrés chrétiens à l'intention des Micmacs appartient donc au protestantisme, au monde anglophone et au siècle suivant. Silas Tertius Rand est né en Nouvelle-Ecosse en 1810, cent ans après Maillard, et y est mort en 1889. Enfant de famille très nombreuse, il est largement l'artisan de sa propre formation. En dehors de quelques brefs séjours au

4. Manuel de Prières, instructions et chants sacrés en hiéroglyphes micmacs / Manual of Prayers, Instructions, Psalms \& Hymns in Micmac Ideograms, New Edition of Father Kauder's Book published in 1866 (Restigouche, The Micmac Messenger, 1921) Centre d'études acadiennes (CEA), cote : 497-K18 N.B. Dans cette réédition réalisée à l'aide du matériel d'impression original, seuls les intertitres allemands ont été remplacés par des indications bilingues français-anglais.

5. Sigentatimgeci, le Cathéchisme Micmac / The Micmac Cathechism (Restigouche, 1913) CEA, cote : 9.5-6.

6. Cf. Dictionnaire biographique du Canada, vol. XI (Québec et Toronto, Les Presses de l'Université Laval, 1982) pp.800-802. 
collège, il étudie surtout en autodidacte, plus ou moins en parallèle avec l'apprentissage du métier paternel de briqueteur; il apprend le latin, le grec, l'hébreu et devient pasteur baptiste en 1834 .

Dans un climat général de ferveur missionnaire envers les populations lointaines, il songe lui-même aux missions étrangères, mais découvre l'existence, sous ses yeux, d'un peuple vivant à l'écart de la civilisation coloniale et de la religion protestante ambiante, et décide en 1846 de consacrer sa vie à l'amélioration du sort spirituel des Micmacs.

Comme Maillard, Rand a eu des précurseurs moins importants. Plusieurs pasteurs protestants, à commencer par l'anglican Thomas Wood, dépositaire, on ne sait pas trop comment, des papiers de Maillard après la mort de celui-ci, ont en effet cherché en vain à arracher les Micmacs à ce qu'ils appelaient les superstitions du papisme. Ces tentatives protestantes s'inscrivent dans le cadre plus général d'un effort politique de pacification et sédentarisation des Amérindiens, perçus comme un peuple à la fois arriéré et dangereux, et semblent diminuer à mesure que les autochtones perdent de leur importance numérique relative et que les difficultés linguistiques s'avèrent insurmontables.

Comme Maillard, Rand peut bénéficier d'une initiation linguistique préliminaire, et partage ensuite la vie des Amérindiens pendant de longues années. Ainsi il acquiert à son tour une connaissance approfondie de leur langue et de leur mode de vie, compose une grammaire et surtout un important dictionnaire anglais-micmac, considéré encore aujourd'hui comme un ouvrage faisant autorite.?

Mais Rand ne peut guère s'appuyer sur un encadrement théologique stable. Le protestantisme dans la colonie anglaise s'est considérablement diversifié et, à côté des baptistes, Rand fait appel à d'autres églises protestantes pour fonder sa Micmac Missionary Society. Il y a certes accord sur les principes de son action combattre le catholicisme et traduire d'urgence les saintes écritures à partir des textes originaux hébreux et grecs - mais les divergences réapparaissent autour de certaines interprétations spécifiques,

7. Silas Rand, Dictionary of the language of the Micmac Indians who reside in Nova Scotia, New Brunswick, Prince Edward Island, Cape Breton and Newfoundland (Halifax, 1888). 
comme la question du baptême. Quand Rand se montre sensible au vœu de la majorité de recourir, comme dans la célèbre Version autorisée anglaise, à un calque du grec «baptizo» en micmac, ses coreligionnaires baptistes, qui pratiquent le baptème des adultes par immersion, s'insurgent: «The Rev. Mr. Rand, engaged in the translation referred to, is well known as a Baptist minister, and as such is expected to give [...] a faithful translation of the word of god (sic) to the Micmac [...] the word «baptizo» means [...] 'to dig, plunge or immerse', and nothing else, and if there is in the language of the Micmac, a word that answers to the greek word 'baptizo', then it is not too much to expect of him, that he will use that word [...] $)^{8}$ Il changera d'ailleurs lui-même d'allégeance religieuse pendant quelque temps avant de revenir à l'église baptiste à la fin de sa vie.

Il reste qu'un dénominateur commun de toutes ces factions protestantes consiste en leur foi dans l'autorité suprême des textes bibliques et dans le pouvoir de persuasion intrinsèque de ceux-ci, pourvu que chacun y ait accès dans sa langue.

Les traductions de Rand sont imprimées à partir de 1853, grâce notamment à la British and Foreign Bible Society, soit en Angleterre, soî à l'Ile-du-Prince-Édouard. Paraissent ainsi les Évangiles selon saint Jean, saint Luc et saint Mathieu, le livre de la Genèse, les Psaumes, les Actes des Apôtres, puis, en 1875 à Halifax, le Nouveau Testament au complet.

Parallèlement, Rand cherche à faciliter l'accès à ses traductions bibliques en enseignant la lecture et l'écriture aux Micmacs qui le souhaitent et en composant pour eux un «Ferst redin buk in Mikmak» pour lequel il utilise une écriture phonétique. Par ailleurs, le pasteur dont l'intérêt linguistique se reflète également dans le nombre de langues qu'il a apprises (douze, dont trois amérindiennes) traduit également en malécite, et il fait imprimer dans cette langue l'Évangile selon saint Jean et quelques passages plus courts, comme les Dix Commandements et le Notre Père.

8. Christian Messenger, August 15, 1851, cité dans Barry Janusas, Silas Rand and the Micmac Missionary Society 1849-1865: the failure of a Protestant mission to the Indians (Halifax, Dalhousie University, Thesis, Honour, 1973). 
Toutefois, son œuvre de traduction biblique, qui est considérable, et son apostolat d'une quarantaine d'années n'ont pas l'impact escompté auprès des destinataires et n'arrivent en tout cas pas à les détacher de la religion catholique.

Il se peut que l'intérêt marqué qu'il manifestait pour l'univers micmac ait contrarié, à la longue, son efficacité de missionnaire. Toujours est-il que les 900 pages manuscrites d'une traduction anglaise des légendes micmaques recueillies, et publiées depuis', qui suscitent encore aujourd'hui l'admiration des anthropologues, témoignent du respect de Rand pour un univers mythologique qui n'est pas le sien. Il y cuvre avec autant de souci de fidélité que dans ses traductions bibliques, selon une méthode de travail en trois étapes: écouter le récit en prenant des notes détaillées, rédiger une version anglaise, et lire - au besoin en retraduisant - ce texte au conteur micmac pour rectification ou approbation.

Comme ailleurs dans l'histoire des civilisations, le passage d'une tradition orale sur le point de disparaître à un texte écrit durable se fait ainsi par la traduction, une traduction à laquelle les récits et légendes des Micmacs doivent non seulement leur entrée dans l'univers des Blancs mais probablement aussi leur survie dans la mémoire des Amérindiens d'aujourd'hui.

\section{Ressemblances et divergences}

Le rapprochement entre Maillard et Rand, les deux grandes figures de la traduction religieuse en langue micmaque avant le $20^{\circ}$ siècle, semble s'imposer. Si les deux hommes se situent de part et d'autre d'une ligne de démarcation religieuse, doublée d'une frontière linguistique, ils se ressemblent en effet sur de nombreux points, dont, en particulier, leur vie auprès des Micmacs pendant de longues années, leur don exceptionnel pour les langues, leur souci de poser d'abord les fondements linguistiques de leurs traductions par une description minutieuse de la grammaire ou du lexique de la langue d'arrivée, leur plaisir évident d'explorer et de traduire, et enfin leur forte personnalité.

9. Silas Tertius Rand, Legends of the Micmacs, Wellesley Philological Publications, (New York and London, Longmans, Green, and Co., 1864). Contient également une bibliographie détaillée des textes publiés et des manuscrits de Rand, pp. XXII-XXIX. 
Certes, à un siècle de distance, ils œuvrent dans des conditions matérielles et intellectuelles quelque peu différentes, mais s'ils divergent dans leur démarche de traducteur, c'est surtout en raison du cadre religieux dans lequel ils accomplissent leur mission.

Maillard s'inscrit dans la tradition catholique du texte sacré dont la lecture intégrale est réservée aux prêtres, médiateurs avertis et spécialement formés. Le message véhiculé par le texte sacré est transmis, et traduit, sous une forme dérivée, telle que fixée dans le catéchisme et par les dogmes. Il n'est donc pas étonnant que les textes traduits par Maillard appartiennent en grande partie à cette dernière catégorie de textes d'interprétation et d'enseignement approuvés qui entourent le texte sacré proprement dit, dont ils finissent par partager certaines caractéristiques.

Rand, pour sa part, est un représentant typique de la foi protestante dans la force du texte sacré lui-même et du culte de la parole de Dieu pour tous. Il traduit par conséquent le Nouveau Testament au complet et, pour l'histoire sainte, les livres de l'Ancien Testament qui relatent les faits historiques. A ce propos il est intéressant de noter que le projet de traduction (jamais mené à bien) de Thomas Wood, anglican et donc membre de l'église protestante la plus proche du catholicisme, se situe quelque part entre les deux, puisqu'il parle de son intention de traduire d'abord le Book of Common Prayer et ensuite la Bible.

Il reste que, sur le plan du choix des textes, les traductions catholiques et protestantes à l'intention des Micmacs font rarement double emploi et contribuent à la constitution d'un corpus important.

Mais il n'y pas que le choix des textes à traduire, il y a aussi les stratégies de traduction qui different. Comme représentant d'une église où les prêtres occupent dans beaucoup de domaines une très forte position d'intermédiaire obligatoire, Maillard semble considérer ses traductions comme un instrument de tutelle. Le choix des hiéroglyphes et la grande attention portée aux effets rhétoriques, à en juger par ses propres remarques et par l'impact de ses traductions, permettent de penser qu'il recherche consciemment la traduction efficace. L'adoption d'un double système d'écriture, justifiée par lui-même comme mesure de sécurité, va dans le même sens.

Étant membre d'une communauté qui n'accorde à ses pasteurs qu'un rôle de guide, Rand adopte une attitude émancipa- 
trice et assimilatrice. Il veut mettre à la disposition des Micmacs le texte sacré lui-même à titre de référence et de recours, et se montre avant tout préoccupé par la possibilité d'accès. D'où l'écriture phonétique uniforme qu'il utilise. Chacun d'eux semble avoir atteint son but sur ce plan, à en juger par l'influence prolongée des cahiers de Maillard sur la vie religieuse des Micmacs, et le rôle d'ouvrage de référence que joue non pas la traduction biblique, certes, mais la collection de légendes de Rand.

\section{Conclusion}

Au-delà des particularités catholiques ou protestantes, on peut dégager de l'œuvre des deux traducteurs trois attitudes fondamentalement différentes devant le texte sacré, et, par voie de conséquence, devant les choix à faire en vue de sa traduction, selon qu'on le considère comme l'objet même de l'effort de mission (Rand, pasteur), ou comme seulement l'un des éléments de la propagation d'une foi qui s'appuie tout autant, sinon davantage, sur des textes de médiation et d'interprétation (Maillard, guide spirituel), ou encore comme un échantillon intéressant des univers mythologiques humains dont l'étude ne vise plus le sacré lui-même, mais les rapports que l'homme entretient avec lui (Rand, anthropologue avant la lettre).

Ainsi, il n'est peut-être pas exagéré de dire que la nature et le sort des traductions de textes sacrés dans le microcosme de la région acadienne et maritime du Canada reflètent assez fídèlement quelques tendances universelles propres à une telle entreprise. Toutefois, l'évocation de l'œuvre micmaque de Maillard et Rand se voulait avant tout l'affirmation d'une expérience canadienne en matière de traduction de textes sacrés, un hommage à deux traducteurs éminents du passé et un rappel de la présence, à nos côtés, des nations amérindiennes du Canada.

Université de Moncton 